\title{
Primeros 100 casos de funduplicatura Nissen asistida por robot en México. Un abordaje que mejorará los resultados de la cirugía antirreflujo. Serie de casos y descripción de la técnica
}

\author{
First 100 cases of robot-assisted Nissen fundoplication in Mexico. \\ An approach that will improve the results of antireflux surgery. \\ Series of cases and description of the technique \\ Javier Alberto Kuri Osorio, ${ }^{*}$ Francisco I Galeana Nogueda, ${ }^{*}$ Karla I Luján Mendoza, ${ }^{*}$ \\ Juan J Solórzano Adame, ${ }^{*}$ Manuel P Aguirre García* \\ * Cirugía General. Cirugía de Mínima Invasión Asistida por Robot. Centro de Cirugía Robótica. Hospital Ángeles Pedregal. Ciudad de México.
}

\section{RESUMEN}

Introducción: El abordaje por mínimo acceso para la cirugía antirreflujo se inicia con el Dr. Dallemagne y colaboradores en 1991. La evolución de la cirugía de mínimo acceso actualmente se encuentra en la cúspide con el abordaje asistido por robot. El inicio de la cirugía robótica en la cirugía antirreflujo se describió en 1999, sólo ocho años después de la cirugía laparoscópica convencional. Los procedimientos asistidos por robot en el área de tubo digestivo alto se han incrementado, hoy en día se puede considerar este abordaje como una herramienta que mejora los resultados de la cirugía antirreflujo. Material y métodos: Análisis retrospectivo de todos los expedientes de pacientes a quienes se les realizó una funduplicatura asistida por robot en el periodo comprendido de enero de 2016 a agosto de 2020 por el mismo equipo quirúrgico. Resultados: Se documentaron 100 casos de funduplicatura en el periodo de estudio, se dividieron los casos en tres grupos: Funduplicaturas primarias 66 (grupo A), refunduplicaturas 28 (grupo B) y refunduplicaturas con piloroplastia seis (grupo C). En el grupo A la edad promedio fue 66.3 años (de 21 a 79 años), 37 fueron mujeres (56\%) y 29 hombres (44\%). El tiempo quirúrgico total fue en promedio de 93.2 minutos (de 60 a 120 minutos) con un tiempo de docking promedio de 8.5 minutos (de seis a 14 minutos). Grupo B la edad promedio fue 63.2 años (de 32 a 78 años), 17 fueron hombres (60\%) y 11 mujeres (40\%). El
ABSTRACT

Introduction: The minimally invasive approach to antireflux surgery begins with Dr. Dallemagne et al. in 1991. The evolution of minimally invasive surgery is currently at the cusp with the robot-assisted approach. The beginning of robotic surgery in antireflux surgery is described in 1999, only eight years after conventional laparoscopic surgery. Robot-assisted procedures in the upper gastrointestinal tract have increased, today this approach can be considered as a tool that improves the results of antireflux surgery. Material and methods: Retrospective analysis of all the records of patients who underwent robot-assisted fundoplication in the period from January 2016 to August 2020 by the same surgical team. Results: 100 cases of fundoplications were documented in the study period, the cases were divided into three groups: Primary fundoplications 66 (group A), re-fundoplications 28 (group B) and re-fundoplications with pyloroplasty 6 (group C). In group $A$, the average age was 66.3 years (21 to 79 years), 37 were women (56\%) and 29 men (44\%). The total surgical time averaged 93.2 minutes (60 to 120 minutes) with an average docking time of 8.5 minutes (6 to 14 minutes). Group B, the average age was 63.2 years (32 to 78 years), 17 were men (60\%) and 11 women (40\%). The total surgical time averaged 124.6 minutes (70 to 190 minutes) with an average docking time of nine minutes (7 to 16 minutes). Group $C$ mean age was 52.7 years (33 to 63 years), four were men (66\%) and

Citar como: Kuri OJA, Galeana NFI, Luján MKI, Solórzano AJJ, Aguirre GMP. Primeros 100 casos de funduplicatura Nissen asistida por robot en México. Un abordaje que mejorará los resultados de la cirugía antirreflujo. Serie de casos y descripción de la técnica. Rev Mex Cir Endoscop. 2020; 21 (2): 71-78. https://dx.doi.org/10.35366/98910 
tiempo quirúrgico total fue en promedio de 124.6 minutos (de 70 a 190 minutos) con un tiempo de docking promedio de nueve minutos (de siete a 16 minutos). Grupo $\mathrm{C}$ la edad promedio fue 52.7 años (de 33 a 63 años), cuatro fueron hombres (66\%) y dos mujeres (34\%). EI tiempo quirúrgico total fue en promedio de 172.1 minutos (de 120 a 210 minutos) con un tiempo de docking promedio de 10 minutos (de siete a 18 minutos). Complicaciones menores transoperatorias en el grupo $\mathrm{A}(1.5 \%)$, complicaciones menores transoperatorias en grupo $\mathrm{B}$ (3.5\%). Reintervenciones, sólo un caso en el grupo A, por migración de la funduplicatura a los tres meses. Grupo $\mathrm{C}$ sin complicaciones. Discusión: En la serie se presenta una morbilidad para REDO (refunduplicatura) de $3.5 \%$ comparada con $2.6 \%$ que se documenta en la literatura internacional. El tiempo quirúrgico total promedio fue de 93.2 minutos para funduplicatura primaria, manteniendo los tiempos quirúrgicos incluso por debajo de los rangos documentados en las series internacionales (de 88 a 137 minutos para funduplicatura primaria). Conclusión: Los resultados hoy en día han desmitificado la cirugía robótica como un procedimiento inalcanzable en costos al tener una reducción en la morbilidad y en los tiempos quirúrgicos. La cirugía robótica en México tiene resultados equiparables a los de la literatura internacional. Se requiere de más estudios en México para obtener mayor relevancia estadística.

Palabras clave: ERGE, cirugía robótica, gastroesofágico, enfermedad por reflujo, funduplicatura, REDO.
2 women (34\%). Total surgical time averaged 172.1 minutes (120 to 210 minutes) with an average docking time of 10 minutes (7 to 18 minutes). Minor intraoperative complications in group A (1.5\%), minor intraoperative complications in group $B(3.5 \%)$. Reinterventions, only one case in group $A$, due to fundoplication migration at three months. Group $C$ without complications. Discussion: The series presents a morbidity for REDO (re-fundoplication) of $3.5 \%$ compared to $2.6 \%$ that is documented in the international literature. The mean total surgical time was 93.2 minutes for primary fundoplication, maintaining surgical times even below the ranges documented in international series (88 to 137 minutes for primary fundoplication). Conclusion: The results today have demystified robotic surgery as an unattainable procedure in costs due to a reduction in morbidity and surgical times. Robotic Surgery in Mexico has results comparable to those of the international literature. More studies are required in Mexico to obtain greater statistical relevance.

Keywords: GERD, gastroesophageal reflux disease, robotic surgery, gastroesophageal, reflux disease, fundoplication, REDO.

\section{INTRODUCCIÓN}

Actualmente la definición de enfermedad por reflujo gastroesofágico (ERGE), más aceptada y además elaborada por un consenso médico, fue descrita por la Organización Mundial de Gastroenterología (WGO) y publicada en el Consenso de Montreal. ERGE se define como una condición que se desarrolla cuando el reflujo del contenido del estómago causa síntomas molestos y/o complicaciones. ${ }^{1}$

En el mundo la enfermedad por reflujo gastroesofágico tiene una prevalencia de $8-33 \% .{ }^{2} \mathrm{El}$ costo de la enfermedad estimado por año es mayor de 9,000-10,000 millones de dólares/año en EUA por el uso de inhibidores de bomba de protones y en pruebas diagnósticas. ${ }^{3}$ En México, la prevalencia de ERGE reportada es de 19.6 a 40\%. ${ }^{4-6}$

ERGE ha sido hasta el día de hoy un padecimiento cuyo objetivo terapéutico es la mejoría de síntomas, los tratamientos médicos continúan a pesar de no obtener un cambio en $100 \%$ de la fisiopatología del reflujo, es decir, sólo se modifica el $\mathrm{pH}$ y hay disminución de los síntomas, pero el reflujo del contenido gástrico persiste en un porcentaje considerable. ${ }^{7}$ En el grupo de estudio LOTUS se demostró una persistencia de la regurgitación de 17.2 y $14.6 \%$ en el seguimiento a seis meses y a cinco años en quienes recibieron tratamiento con esomeprazol, comparado con 8.5 y $0.9 \%$ en el grupo de cirugía antirreflujo ( $p<0.001$ ), de igual forma en este estudio se demuestra una reducción en el tiempo de exposición del esófago a un $\mathrm{pH}<4$ en el grupo de pacientes con cirugía antirreflujo en comparación con el tratamiento médico en un seguimiento de cinco años con datos estadísticamente significativos $(p<0.001){ }^{8}$

ERGE es un padecimiento que sigue teniendo indicaciones quirúrgicas basadas en un problema sintomático y en una evaluación de calidad de vida por los síntomas; sin embargo, actualmente ERGE es el factor de riesgo con mayor asociación al esófago de Barrett y al cáncer de esófago, patología que registra $5.3 \%$ de las muertes por cáncer de acuerdo al GLOBOCAN de 2018.9-11

En 1991 el abordaje de mínimo acceso para la cirugía antirreflujo se inicia con el Dr. Dallemagne y colaboradores. ${ }^{12}$ La evolución de la cirugía de mínimo acceso actualmente se encuentra en la cúspide con el abordaje asistido por robot. El inicio de la cirugía robótica en la cirugía antirreflujo se describió en 1999, sólo ocho años después de la cirugía laparoscópica convencional. ${ }^{13}$

La cirugía robótica con la plataforma en modalidad de cirugía robot esclavo llegó a México (sistema quirúrgico Da Vinci) en 2006 realizando el primer procedimiento bariátrico y urológico asistido por robot. ${ }^{14,15}$ Después de 14 años los procedimientos asistidos por robot en el área de tubo digestivo alto se han incrementado, hoy en día se puede considerar este abordaje como una herramienta que mejora los resultados de la cirugía antirreflujo.

El objetivo de este artículo es presentar la experiencia inicial de un solo grupo quirúrgico en México así como la descripción de la técnica quirúrgica. 


\section{MATERIAL Y MÉTODOS}

Éste es un estudio observacional y retrospectivo que tiene como objetivo reportar la experiencia obtenida en las primeras 100 funduplicaturas realizadas por un grupo quirúrgico experto en abordaje asistido por robot gastrointestinal. Se analizan los datos de los expedientes de pacientes a quienes se les efectuó una funduplicatura asistida por robot en el periodo comprendido de enero de 2016 a agosto de 2020. El cirujano titular realizó todos los procedimientos asistido por dos cirujanos con entrenamiento en cirugía robótica. Todos los pacientes que se incluyen en la revisión cumplían con los criterios de SAGES ${ }^{16}$ y con la definición de Montreal para enfermedad por reflujo gastroesofágico. ${ }^{1}$

A todos los pacientes se les realizó fisiología esofágica mediante manometría de alta resolución en el protocolo preoperatorio para descartar alteraciones en la motilidad esofágica. En todos los pacientes se aplicó un procedimiento de funduplicatura tipo Nissen asistido por robot con endoscopía transoperatoria bajo anestesia general balanceada con bloqueo neuromuscular profundo, el paciente se colocó en decúbito supino, el neumoperitoneo se abordó por técnica de Veress a $12 \mathrm{mmHg}$ a través de la cicatriz umbilical. Los trocares se colocan de acuerdo con la filosofía de puertos del sistema quirúrgico Da Vinci SI ${ }^{\circledR}$ (Figura 1), se realizan las medidas del sitio anatómico quirúrgico (surgical target) con la cavidad peritoneal insuflada. Se coloca marcaje para puerto de cámara a $20 \mathrm{~cm}$ caudales al hiato esofágico, localizado $9 \mathrm{~cm}$ por arriba del apéndice xifoides y se coloca trocar de $12 \mathrm{~mm}$. Los trocares de 8 $\mathrm{mm}$ para brazo 1 y 2 robótico se colocan $8-10 \mathrm{~cm}$ laterales hacia la derecha y a la izquierda del trocar de la cámara, separador de Nathanson colocado subxifoides de $5 \mathrm{~mm}$, trocar auxiliar de $5 \mathrm{~mm}$ lateral al trocar de $8 \mathrm{~mm}$ del lado izquierdo (sobre la línea axilar anterior) para retracción y aspiración. El docking (acoplamiento) se realizó de forma lateral (side docking) y sólo se utilizaron dos brazos robóticos de trabajo con pinzas: ProGrasp ${ }^{\mathrm{TM}}$, portaagujas con corte e inserto armónico.

Se realizó el procedimiento de funduplicatura primaria con la siguiente técnica:

- Disección de la pars flácida hasta identificar el pilar derecho del hiato esofágico (Figura 2).

- Disección periesofágica de la membrana frenoesofágica (Figura 3).

- Ventana retroesofágica y colocación de Penrose para tracción caudal de esófago (Figura 4).

- Disección periesofágica proximal (Figura 5).

- Liberación de vasos cortos del fundus gástrico hasta el ángulo de His con inserto armónico. (Figura 6).

- Cierre de pilares del hiato con poliéster 2.0 (Figura 7).

- Funduplicatura de $360^{\circ}$ esófago-fundus-esófago con poliéster 2.0, longitud de $2.5-3 \mathrm{~cm}$, calibrada con Maloney $56 \mathrm{Fr}$ (Figura 8).

- Gastropexia anterolateral de la curvatura mayor a la pared abdominal con punto de poliéster 2.0 (Figura 9).

- Endoscopía transoperatoria corroborando morfología de funduplicatura en retroversión y con estómago insuflado.

El procedimiento de REDO (refunduplicatura) se realiza siguiendo los mismos pasos de la cirugía de funduplicatura primaria, agregando en un inicio los siguientes pasos:

Figura 1:

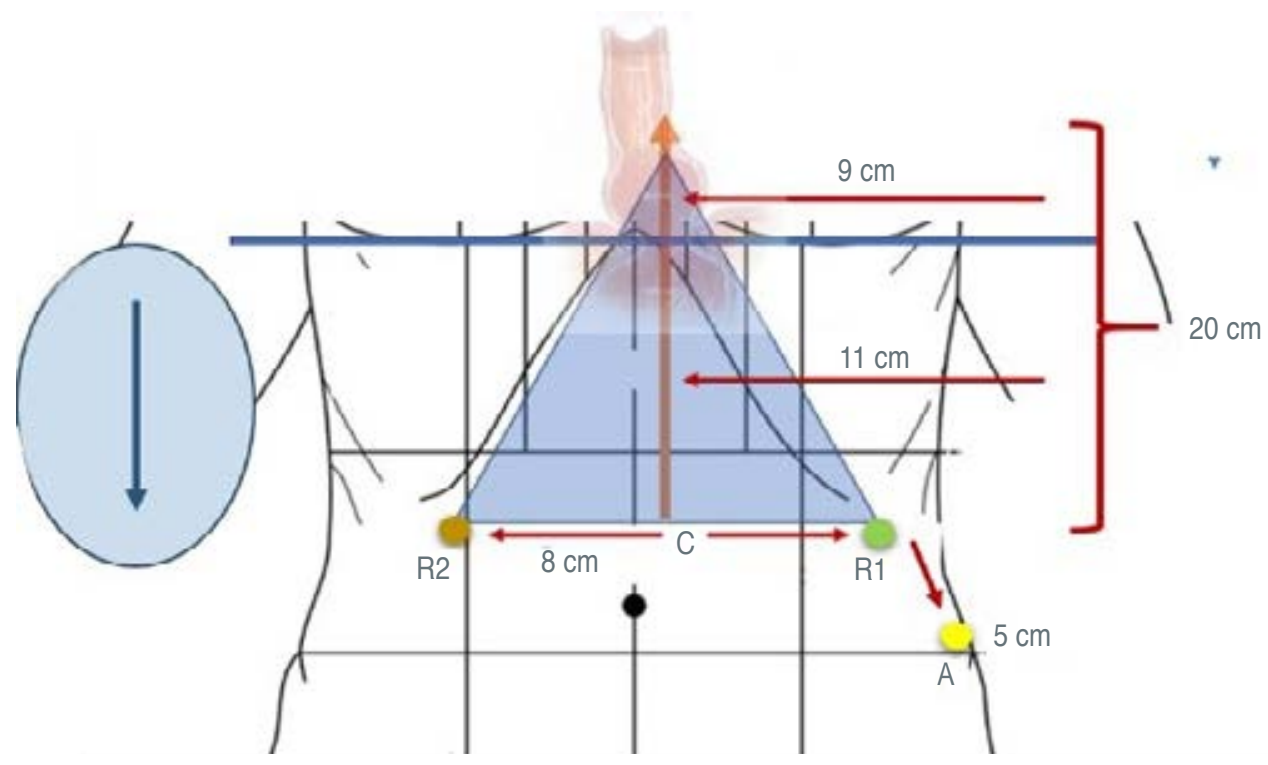

Filosofía de puertos del sistema quirúrgico Da Vinci $\mathrm{SI}^{\circledR}$. 


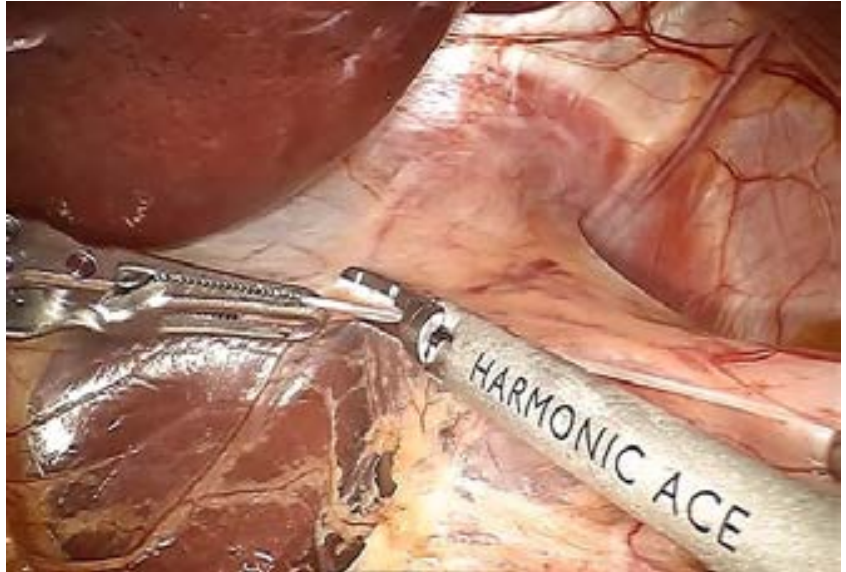

Figura 2: Disección de la pars flácida hasta identificar el pilar derecho del hiato esofágico.

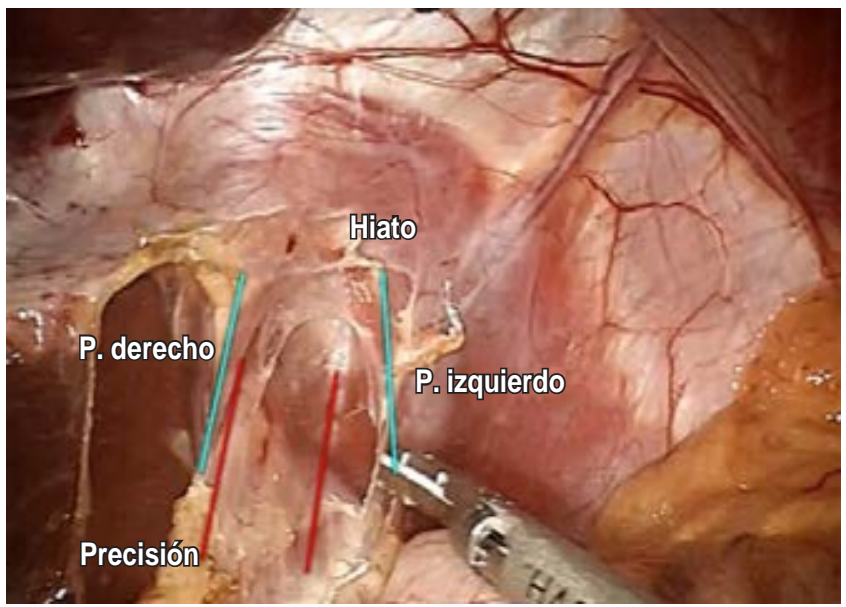

Figura 3: Disección periesofágica de la membrana frenoesofágica

- Liberación de las adherencias al hiato por el procedimiento previo.

- Desmantelamiento de la funduplicatura previa mediante disección roma y energía con inserto armónico.

- Se realiza nuevamente funduplicatura tipo Nissen, de $2.5-3 \mathrm{~cm}$ de longitud con poliéster 2.0, fundusesófago-fundus, Maloney 56Fr como calibrador.

- Gastropexia anterolateral a pared abdominal con poliéster 2.0.

- El procedimiento de piloroplastia se realizó con técnica de Heineke-Mikulicz utilizando sutura barbada 2.0.

- Endoscopía transoperatoria para evaluación de la morfología de la funduplicatura y competencia de la misma con estómago insuflado.
Los pacientes del estudio se agruparon de la siguiente manera:

- Grupo A: Pacientes con diagnóstico de enfermedad por reflujo gastroesofágico de acuerdo a las guías de SAGES y a la definición de Montreal, con endoscopía y manometría preoperatoria. A los pacientes con esofagitis por endoscopía sólo se les realizó manometría y no pHmetría, ya que el diagnóstico por pHmetría se efectúa por exclusión cuando los hallazgos clínicos de ERGE no corresponden a los hallazgos endoscópicos.

- Grupo B: Pacientes con persistencia de sintomatología de reflujo. Se confirmó el diagnóstico de falla en funduplicatura previa para manejo de

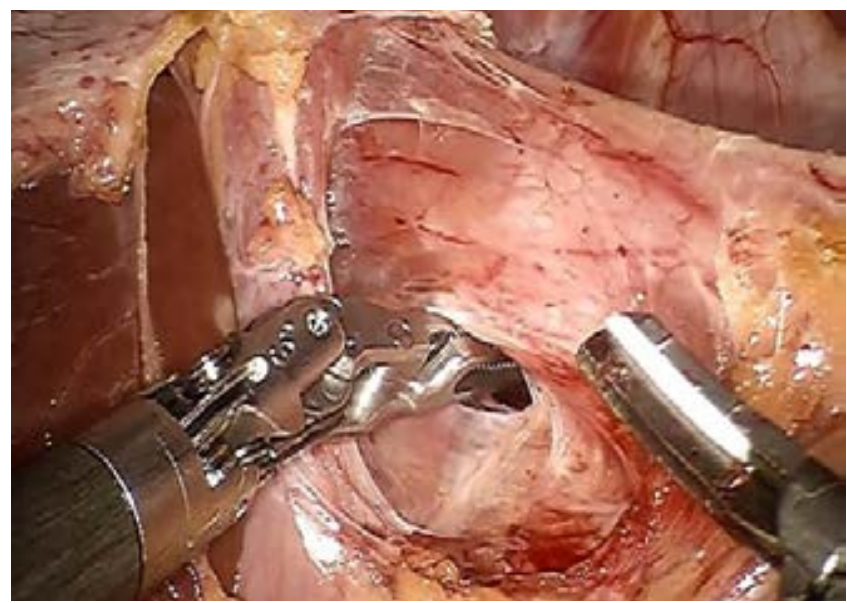

Figura 4: Ventana retroesofágica y colocación de Penrose para tracción caudal de esófago.

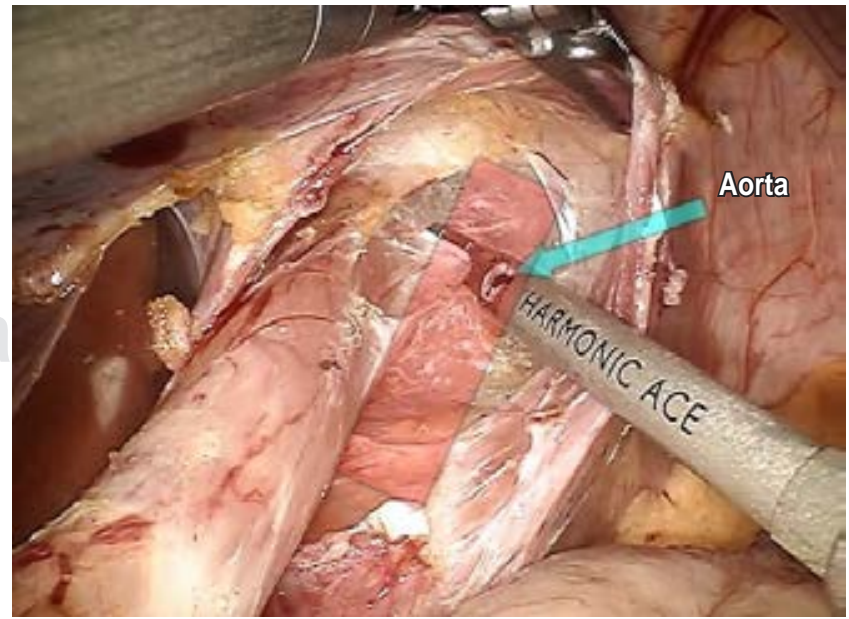

Figura 5: Disección periesofágica proximal. 


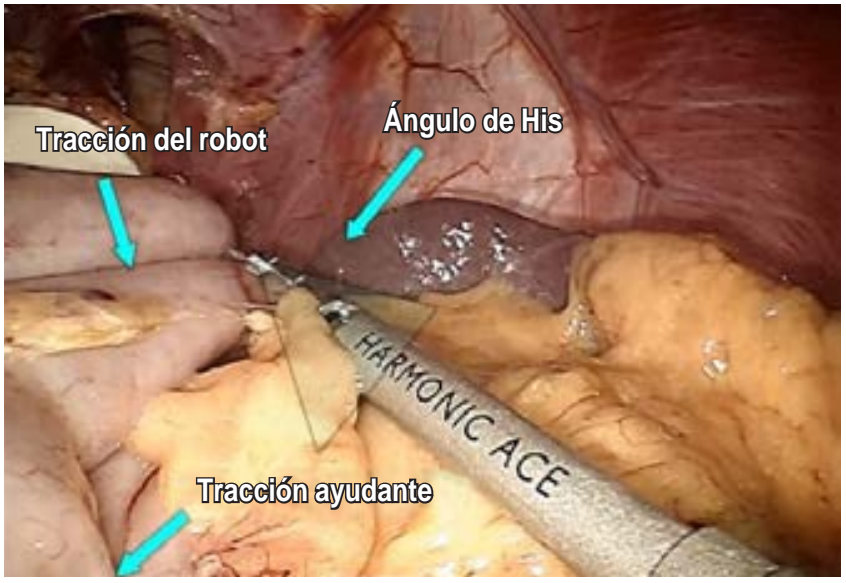

Figura 6: Liberación de vasos cortos del fundus gástrico hasta el ángulo de His con inserto armónico.

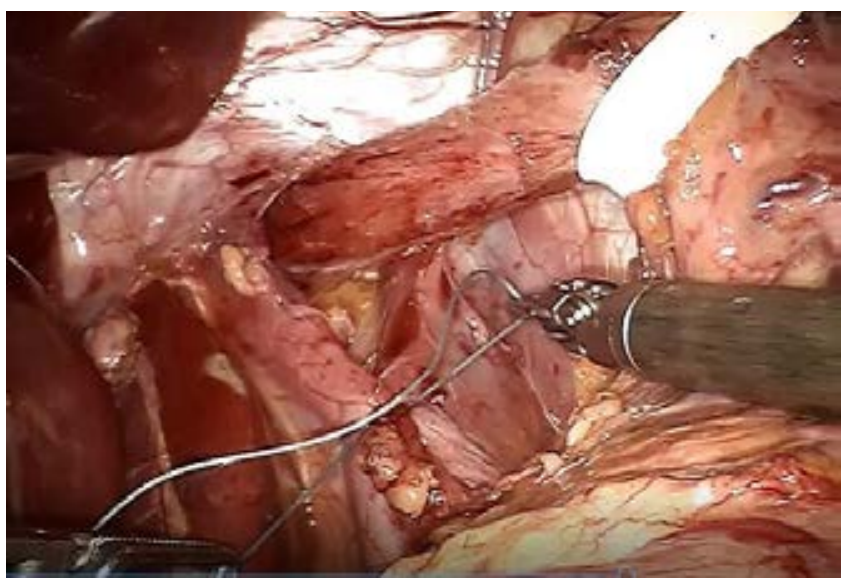

Figura 7: Cierre de pilares del hiato con poliéster 2.0.

ERGE mediante esofagograma y/o endoscopía de tubo digestivo alto. La indicación de manejo quirúrgico fue el impacto severo en la calidad de vida de los pacientes por persistencia de síntomas de reflujo gastroesofágico.

- Grupo C: Pacientes con persistencia de sintomatología de reflujo, se confirmó el diagnóstico de falla en funduplicatura previa para manejo de ERGE mediante esofagograma y/o endoscopía de tubo digestivo alto con hallazgos endoscópicos de retraso en el vaciamiento gástrico (presencia de restos alimenticios en un periodo de ayuno mayor de cuatro horas) que se confirmó por gammagrama gástrico. El retraso en el vaciamiento gástrico es promotor de persistencia de ERGE, por lo que se realizó funduplicatura + piloroplastia como manejo quirúrgico para este grupo de pacientes. ${ }^{17}$
Se obtuvieron las siguientes variables de tipo cuantitativo de los registros posquirúrgicos del expediente clínico: Tiempo quirúrgico, sangrado, tiempo de docking. La edad y el sexo se consideraron datos demográficos.

\section{RESULTADOS}

Se documentaron 100 casos de funduplicaturas en el periodo de estudio, se dividieron los casos en tres grupos: Funduplicaturas primarias 66 (grupo A), refunduplicaturas 28 (grupo B) y refunduplicaturas con piloroplastia seis (grupo C) (Tabla 1).

En el grupo A la edad promedio fue 66.3 años (de 21 a 79 años), 37 fueron mujeres (56\%) y 29 hombres (44\%). El tiempo quirúrgico total fue en promedio de 93.2 minutos (de 60 a 120 minutos) con un tiempo de docking prome-

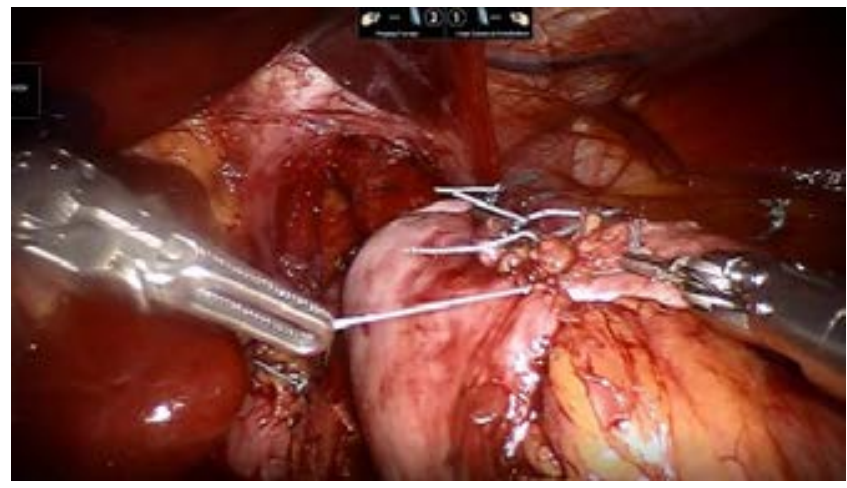

Figura 8: Funduplicatura de $360^{\circ}$ esófago-fundus-esófago con poliéster 2.0, longitud de 2.5-3cm, calibrada con Maloney $56 \mathrm{Fr}$.

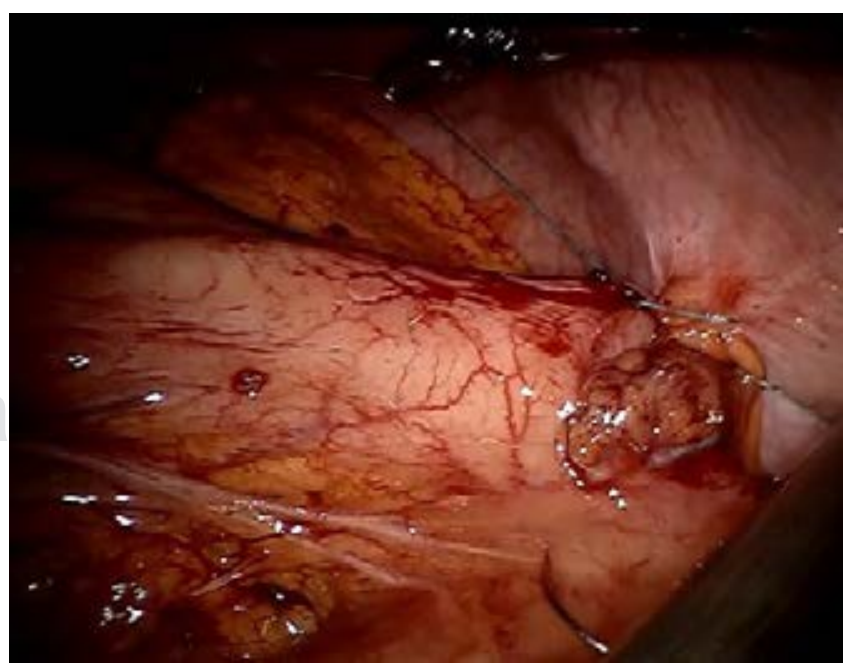

Figura 9: Gastropexia anterolateral de la curvatura mayor a la pared abdominal con punto de poliéster 2.0. 
Tabla 1: Descripción de los resultados por grupo quirúrgico.

\begin{tabular}{lccc} 
& Grupo A & Grupo B & $\begin{array}{c}\text { Grupo C } \\
(n=6) \\
(n=66) \\
n(\%)\end{array}$ \\
Hombres & $n(\%)$ & 17 & 4 \\
Mujeres & 29 & 11 & 2 \\
Edad, años (promedio) & 37 & $63.2(32-78)$ & $52.7(33-63)$ \\
Sangrado (mL) & $66.3(21-79)$ & 15 & 15 \\
Tiempo de docking, min (promedio) & Mínimo & $9(7-16)$ & $10(8-18)$ \\
Tiempo quirúrgico total, min (promedio) & $8.5(6-14)$ & $124.6(70-190)$ & $172.1(120-210)$ \\
\hline
\end{tabular}

dio de 8.5 minutos (de 6 a 14 minutos). Se presentaron dos complicaciones:

- Transoperatorias: Un neumotórax en un paciente de 47 años con hernia de hiato de $9 \mathrm{~cm}$ con contenido de fundus gástrico en el saco herniario, en el momento de la reducción del saco herniario se lesionó la pleura izquierda, se resolvió al término del procedimiento con punción con catéter 14G y Valsalva previo a la extubación del paciente y no requirió sonda endopleural.

- Posoperatorias: En una mujer de 41 años quien reingresó a los tres meses por migración de funduplicatura secundaria a esfuerzo por vómito en una intoxicación alimentaria, se resolvió con cirugía asistida por robot, reduciendo funduplicatura y gastropexia.

En el grupo B la edad promedio fue 63.2 años (de 32 a 78 años), 17 fueron hombres (60\%) y 11 mujeres (40\%). El tiempo quirúrgico total fue en promedio de $124.6 \mathrm{mi}-$ nutos (de 70 a 190 minutos) con un tiempo de docking promedio de nueve minutos (de siete a 16 minutos). Se presentó una complicación transoperatoria: Un neumotórax izquierdo durante la disección de la funduplicatura migrada se resolvió con el mismo procedimiento utilizado en el neumotórax del grupo A.

En el grupo $\mathrm{C}$ la edad promedio fue 52.7 años (de 33 a 63 años), cuatro fueron hombres (66\%) y dos mujeres (34\%). El tiempo quirúrgico total fue en promedio de 172.1 minutos (de 120 a 210 minutos) con un tiempo de docking promedio de 10 minutos (de siete a 18 minutos). No se documentaron complicaciones en este grupo.

\section{DISCUSIÓN}

El abordaje de mínimo acceso para la cirugía antirreflujo se ha consolidado hoy en día como un estándar de oro para el manejo de la enfermedad por reflujo gastroesofágico, lo anterior se fundamenta en los beneficios demostrados en corta estancia hospitalaria y menor tasa de complicaciones en periodo perioperatorio así como buenos resultados en la disminución de la exposición de la mucosa esofágica al jugo gástrico a corto y largo plazo. ${ }^{18}$

El abordaje asistido por robot para realizar una funduplicatura empieza a reflejar resultados superiores a los obtenidos por laparoscopía. En 2012 Marzio Frazzoni y colaboradores en un estudio de 44 pacientes en el grupo laparoscópico y 44 en el grupo robótico documentan mayor disminución de valores anormales del tiempo de exposición esofágica al ácido al ser comparado con el abordaje laparoscópico convencional, $14 \%$ de los casos en el grupo laparoscópico comparado con cero casos en el grupo robótico. ${ }^{19}$

El procedimiento de tipo REDO se describe en la literatura médica como un procedimiento con alta morbilidad perioperatoria (7.3-9.9\%), ${ }^{20,21}$ el abordaje asistido por robot permite al cirujano mayor precisión en el manejo de los tejidos ya modificados por los procedimientos previos disminuyendo la morbilidad perioperatoria asociada a la cirugía. Alexander C. Mertens y Rob C. Tolboom en una revisión retrospectiva de 151 procedimientos de REDO documentan una incidencia de $2.6 \%$ para complicaciones mayores en el periodo perioperatorio. ${ }^{22}$ En nuestra serie se documentó sólo una complicación no grave $(3.5 \%)$ en el grupo de REDO que se resolvió sin reintervención quirúrgica.

El tiempo quirúrgico de una funduplicatura primaria asistida por robot se ha documentado entre 88 y $137 \mathrm{mi}-$ nutos. ${ }^{23}$ En nuestra serie el tiempo para un procedimiento primario fue de 60 a 120 minutos (promedio de $93.2 \mathrm{mi}$ nutos) encontrándose dentro de los tiempos que manejan los centros internacionales de referencia y alta especialidad. Es importante mencionar que el acoplamiento lateral (side docking) permite un libre acceso a la vía aérea y al control endoscópico que se realiza en el transoperatorio. 
La gastroparesia en pacientes con antecedentes de funduplicatura es un padecimiento que cada vez se asocia con mayor frecuencia, teniendo como principal factor de riesgo la lesión en nervios vagos durante un procedimiento de cirugía gástrica. ${ }^{24,25}$ Los síntomas generados por un retraso en el vaciamiento gástrico en un paciente con funduplicatura son de gran impacto en la calidad de vida, siendo la plenitud posprandial, náusea y distensión gástrica los principales, por lo tanto la realización de piloroplastia en un contexto de funduplicatura fallida con diagnóstico de retraso en el vaciamiento gástrico mejora notablemente la resolución de síntomas y la calidad de vida posoperatoria. ${ }^{26}$

\section{CONCLUSIONES}

El abordaje asistido por robot para realizar una funduplicatura es la evolución de un procedimiento de mínimo acceso en el que se superan los obstáculos del abordaje laparoscópico convencional como los rangos de movimiento de las pinzas robóticas en ángulos a nivel del hiato esofágico, logrando efectuar una disección y manipulación de los tejidos con mayor precisión y mejorando la definición de la mano del cirujano; sin embargo, se requiere de más estudios para demostrar estos resultados.

Con el objetivo de tener una referencia con una evidencia estadística significativa en México, el grupo quirúrgico que dirige esta publicación se encuentra elaborando un estudio prospectivo con los resultados.

\section{REFERENCIAS}

1. Vakil N, Van Zanten SV, Kahrilas P et al. The Montreal definition and classification of gastroesophageal reflux disease: a global evidence-based consensus. Am J Gastroenterol. 2006; 101: 1900-20. quiz 1943.

2. El-Serag HB, Sweet S, Winchester CC et al. Update on the epidemiology of gastrooesophageal reflux disease: a systematic review. Gut. 2014; 63: 871-880.

3. Shaheen NJ, Hansen RA, Morgan DR et al. The burden of gastrointestinal and liver diseases, 2006. Am J Gastroenterol. 2006; 101: 2128-2138.

4. López-Colombo A, Morgan D, Bravo-González D et al. The epidemiology of functional gastrointestinal disorders in Mexico: a population-based study. Gastroenterol Res Pract. 2012; 2012: 606174.

5. Schmulson M. Presencia de pirosis según Roma II en Español-México; obliga a descartar reflujo gastroesofágico. Rev Gastroenterol Mex. 2009; 74: 74-76.

6. Huerta-Iga F. Síntomas gastrointestinales en México. Un estudio epidemiológico SIGAME. Remes-Troche JM. ASECOM Editorial, S.A. de C.V. 2015, p. 29-42.

7. Tamhankar AP, Peters JH, Portale G et al. Omeprazole does not reduce gastroesophageal reflux: New insights using multichannel intraluminal impedance technology. J Gastrointest Surg. 2004; 8: 890.

8. Hatlebakk JG, Zerbib F, Bruley des Varannes $S$ et al. Gastroesophageal acid reflux control 5 years after antireflux surgery, compared with long-term esomeprazole therapy. Clin Gastroenterol Hepatol. 2016; 14 (5): 678-685.e3. doi: 10.1016/j.cgh.2015.07.025.

9. Coleman HG, Xie SH, Lagergren J. The epidemiology of esophageal adenocarcinoma. Gastroenterology. 2018; 154: 390-405.

10. Cook MB, Corley DA, Murray LJ, Liao LM, Kamangar F, Ye W, Gammon MD et al. Gastroesophageal reflux in relation to adenocarcinomas of the esophagus: a pooled analysis from the Barrett's and esophageal adenocarcinoma consortium (BEACON). PLoS One. 2014; 9: e103508.

11. Bray F, Ferlay J, Soerjomataram I, Siegel RL, Torre LA, Jemal A. Global cancer statistics 2018: GLOBOCAN estimates of incidence and mortality worldwide for 36 cancers in 185 countries. CA Cancer J Clin. 2018; 68 (6): 394-424. doi: 10.3322/caac.21492.

12. Dallemagne B, Weerts JM, Jehaes C, Markiewicz S, Lombard R. Laparoscopic Nissen fundoplication: preliminary report. Surg Laparosc Endosc. 1991; 1 (3): 138-143.

13. Cadiere GB, Himpens J, Vertruyen M, Bruyns J, Fourtanier G. Nissen fundoplication done by remotely controlled robotic technique. Ann Chir. 1999; 53 (2): 137-141.

14. González RR, Ballí JJ, Rumbaut RD. Nuevas tecnologías en cirugía: cirugía robótica. Avances. 2007; 13: 41-50.

15. Corona-Montes VE. La cirugía robótica como alternativa para el tratamiento del cáncer de próstata de bajo riesgo en México. Rev Mex Urol. 2013; 73: 221-222.

16. Stefanidis D, Hope WW, Kohn GP et al. Guidelines for surgical treatment of gastroesophageal reflux disease. Surg Endosc. 2010; 24 (11): 2647-2669. doi: 10.1007/s $/ \mathrm{s}^{0} 0464-$ 010-1267-8. SAGES 2010.

17. Masqusi S, Velanovich V. Pyloroplasty with fundoplication in the treatment of combined gastroesophageal reflux disease and bloating. World J Surg. 2007; 31 (2): 332-336. doi: 10.1007/s $0268-006-0723-z$.

18. Qu H, Liu Y, He QS. Short- and long-term results of laparoscopic versus open anti-reflux surgery: a systematic review and meta-analysis of randomized controlled trials. J Gastrointest Surg. 2014; 18 (6): 1077-1086. doi: 10.1007/ s'1605-014-2492-6.

19. Frazzoni M, Conigliaro R, Colli G, Melotti G. Conventional versus robot-assisted laparoscopic Nissen fundoplication: a comparison of postoperative acid reflux parameters. Surg Endosc. 2012; 26 (6): 1675-1681. doi: 10.1007/s $0464-$ 011-2091-5.

20. Nageswaran H, Haque A, Zia M, Hassn A. Laparoscopic redo anti-reflux surgery: Case-series of different presentations, varied management and their outcomes. Int J Surg. 2017; 46: 47-52. doi: 10.1016/j. ijsu.2017.08.553.

21. Singhal S, Kirkpatrick DR, Masuda T, Gerhardt J, Mittal SK. Primary and redo antireflux surgery: outcomes and lessons 
learned. J Gastrointest Surg. 2018; 22 (2): 177-186. doi: 10.1007/s $1605-017-3480-4$.

22. Mertens AC, Tolboom RC, Zavrtanik H, Draaisma WA, Broeders IAMJ. Morbidity and mortality in complex robotassisted hiatal hernia surgery: 7-year experience in a highvolume center. Surg Endosc. 2019; 33 (7): 2152-2161. doi: 10.1007/s $\mathrm{s}^{0}$ 0464-018-6494-4.

23. Zhang P, Tian JH, Yang KH et al. Robot-assisted laparoscope fundoplication for gastroesophageal reflux disease: a systematic review of randomized controlled trials. Digestion. 2010; 81 (1): 1-9. doi: 10.1159/000235920.
24. Soykan I, Sivri B, Kiernan B, NcCallum RW. Demography, clinical characteristics, psychological and abuse profiles treatment, and long-term follow-up of patients with gastroparesis. Dig Dis Sci. 1998; 43 (11): 2398-2404.

25. Shafi MA, Pasricha PJ. Post-surgical and obstructive gastroparesis. Curr Gastroenterol Rep. 2007; 9: 280. https ://doi.org/10.1007/s¹89 4-007-0031-2.

26. Khajanchee YS, Dunst CM, Swanstrom LL. Outcomes of Nissen fundoplication in patients with gastroesophageal reflux disease and delayed gastric emptying. Arch Surg. 2009; 144 (9): 823-828. doi: 10.1001/archsurg.2009.160. 